\title{
Analysis of different digital filters for received signal strength indicator
}

\author{
Rafhanah Shazwani Rosli, Mohamed Hadi Habaebi, Md. Rafiqul Islam \\ Department of Electrical and Computer Engineering, International Islamic University Malaysia, Malaysia
}

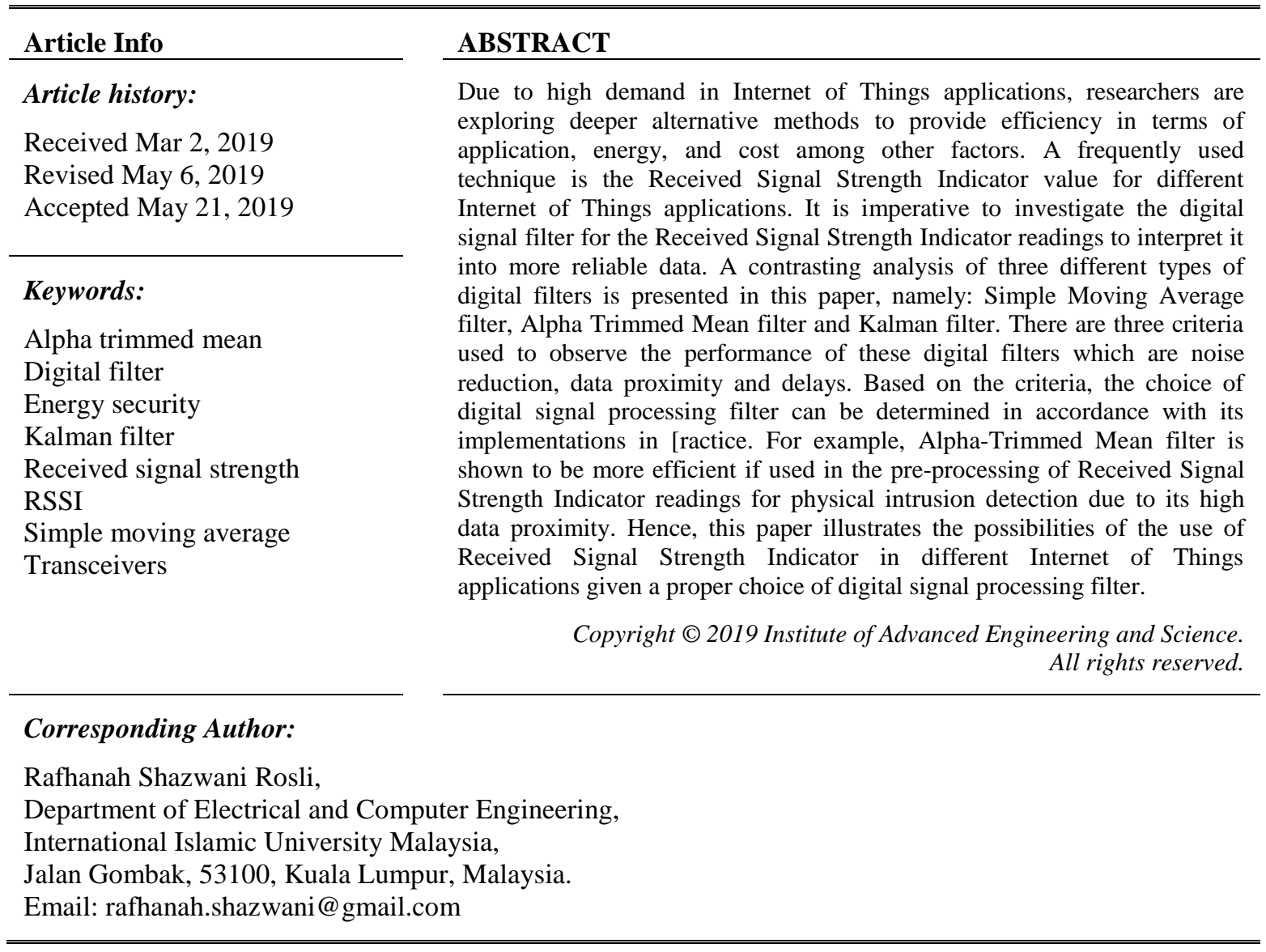

\section{INTRODUCTION}

One of the parameters that is widely known in the area of wireless networking environment is Received Signal Strength Indicator (RSSI). In the IEEE 802.11 system, RSSI is the approximate strength of received signal in arbitrary units. The value of RSSI can be interpreted by voltage changes processing as measured by the circuit of a receiver [1]. However, IEEE 802.11 standards have not defined RSSI readings to be related with any standard physical parameter. The readings of RSSI are correlated with the distance between the transmitter and receiver. There are several factors that may affect the RSSI value perceived by the receiver. Some of the factors are reflection, diffraction, signal source and also obstruction in the path of the radio signal wave [2]. Furthermore, the human body can also cause attenuation on the wireless signal. Attenuation of the wireless signal is reflected on the reading of RSSI value received.

Numerous studies have discussed RSSI, its characteristics and implementations. One of the implementations of RSSI is in indoor tracking and positioning which are discussed in [3-5]. Furthermore, RSSI is also implemented for localization scenario and location-aware system as discussed in [6-10]. Apart from RSSI, there are several different techniques that can be used such as Angle of Arrival, Time of Arrival and Time Difference of Arrival. However, with the use of the RSSI technique, location estimated systems can be developed without the use of a specialized hardware. The implementation of RSSI for indoor 
localization brings up some issues that should be overcome such as signal attenuation caused by obstructions, multipath fading effect and other noises that affect RSSI readings.

Hence, the objective of this paper is to compare and analyze a few types of digital filters that can be used for the processing of RSSI readings which can help to interpret it into more reliable data for different Internet of Things (IoT) related implementations. This paper is an extensive work as presented in [11]. This paper is also correlated to the work as presented in [12]. The organization of this paper is in the following manner. Section 1 is the Introduction that briefly explains the general background of RSSI, related researches and the aim of this paper. Section 2 explains the experimental setup and method used to analyze the different type of digital filters used on the RSSI readings. Section 3 presents the results obtained from experimentations along with its discussion. Section 4 concludes the paper. Lastly, acknowledgment, references and biographies of authors are included in the last three sections.

\section{RESEARCH METHOD}

The digital filters for RSSI readings are analyzed and compared by conducting experiments using ESP8266 WiFi serial transceiver module. It is a self-contained system on a chip integrated with TCP/IP protocol stack, WiFi direct Peer-to-Peer (P2P) and soft Access Point (AP) mode. It supports APSD for VoIP application and also Bluetooth co-existence interfaces. ESP8266 is also equipped with a self-calibrated RF [13]. There are various models of the ESP8266 WiFi serial transceiver module. The most basic and general version, which is ESP8266-01 is sufficient to be used for the analysis in this paper. Figure 1 shows the ESP8266-01 model with its pins distribution and size measurement. Through its general-purpose input/output (GPIO) pins, the module is able to be integrated with sensors or any other application specific devices. It has an onboard processing and storage capability enabling it to be integrated with sensors or many other devices for the Internet of Things application.

The ESP8266 WiFi serial transceiver module is programmed with the algorithm to behave as either transmitter or receiver. The transmitter ESP8266 is programmed to act as an access point that has a unique SSID and password. The receiver ESP8266 is programmed to connect to the WiFi Protected Access 2 (WPA2) network of the transmitter ESP8266 and read its RSSI value. There are three types of digital filters used in the experiment of this paper which are Simple Moving Average (SMA), Alpha Trimmed Mean (ATM) and Kalman Algorithm. The algorithm of each filter is programmed into the receiver ESP8266 to produce its filtered RSSI reading values. The values are then portrayed into graphs that are presented in Section 3.

Figure 2 shows the experimental setup and layout to investigate the effect of different digital filters to RSSI readings. The transmitter and receiver are positioned $1 \mathrm{~m}$ above the ground and $1 \mathrm{~m}$ apart from each other. They are located in between a pathway to let a human subject cross the LoS between the transmitter and receiver. The same human subject is used throughout the experiment. The crossing of a human subject is included in the experiment condition in order to observe the noises produced.

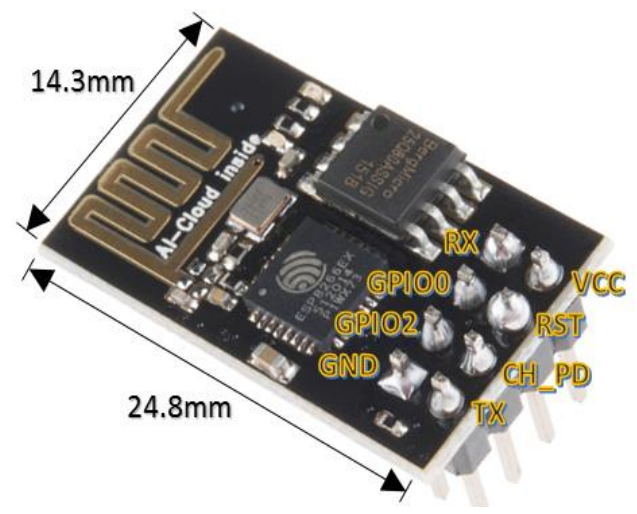

Figure 1. ESP8266-01 pins distribution and size measurement

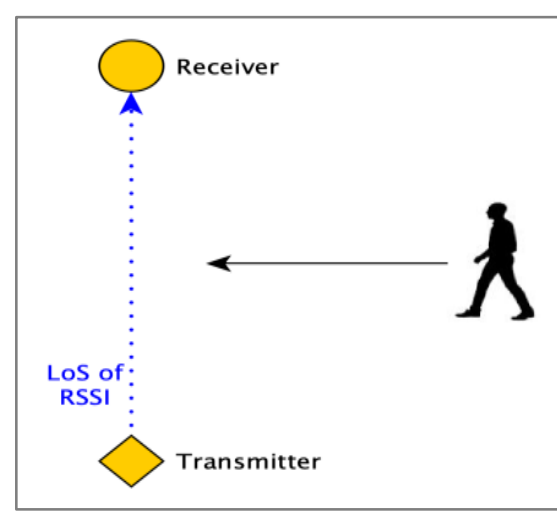

Figure 2. Experimental setup and layout [10] 


\subsection{Simple Moving Average}

Simple Moving Average (SMA) is a simple low pass Finite Impulse Response (FIR). It is suitable to be used on RSSI readings due to its simplicity and efficiency. SMA comes in handy to filter unwanted noise from the raw RSSI data. SMA works by taking n readings of RSSI and calculating its average to produce a single averaged reading of RSSI. In this paper, the parameter is set at $n=5$. The SMA algorithm can be expressed in the equation 1 as follows:

$$
Y[i]=\frac{1}{n} \sum_{j=0}^{n-1} X[i+j]
$$

where; $Y[]=$ Filtered RSSI value

$X[]=$ Raw RSSI values

$n=$ Number of readings

\subsection{Alpha trimmed mean}

Alpha Trimmed Mean (ATM) is one of the suitable digital filters to be used in the pre-processing of RSSI readings. ATM filters unwanted noises especially random spikes. The algorithm of ATM is portrayed in the flowchart of Figure 3. The algorithm starts by initializing the window size, $n$ and alpha, $\alpha$. Suitable parameter can be determined through offline testing phases according to the implementation of RSSI readings. In this paper, the parameters are set at $n=5$ and $\alpha=2$. The array of size $n$ is filled with raw RSSI readings. The raw RSSI readings are then sorted from smallest to largest or vice versa. The algorithm proceeds with discarding $\alpha / 2$ elements from each end of the array. The value of alpha should be as in equation 2 . The filtered RSSI value is obtained by averaging the remaining elements in the array by $n-\alpha$.

$$
\text { Alpha, } \alpha<\frac{1}{2}(\text { Window Size, } n)
$$

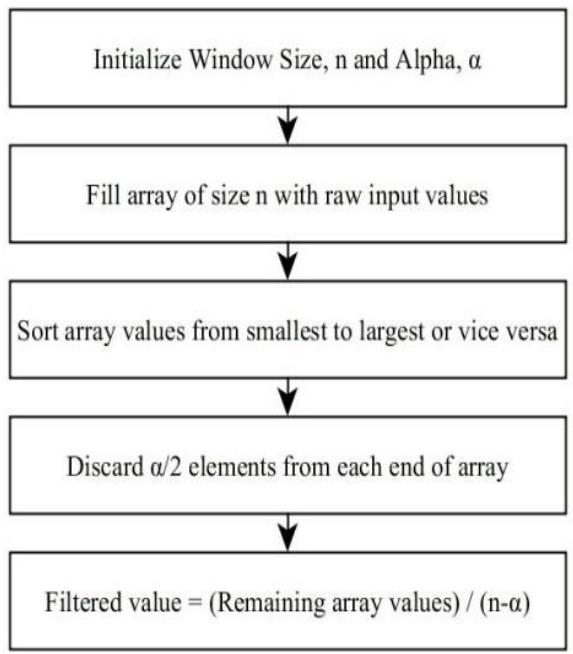

Figure 3. Algorithm of Alpha Trimmed Mean digital filter

\subsection{Kalman}

Kalman filter is an exceptional pre-processing tool that significantly removes noises from raw RSSI readings. It is a recursive algorithm that takes the previous RSSI readings into account. A regular Kalman filter that assumes linear models is sufficient as RSSI reading is a static system. The equations for computing Kalman filter is defined by (3) to (7) as follows [14, 15]:

Prediction step:

$$
\begin{aligned}
& \bar{x}=x \\
& p=p+Q
\end{aligned}
$$


Measurement update:

$$
\begin{aligned}
& k=\frac{p}{(p+R)} \\
& x=\bar{x}+k(R S S I-\bar{x}) \\
& p=p(1-k)
\end{aligned}
$$

where; $x=$ Filtered RSSI value

$p=$ Estimated error

$Q=$ Process noise

$R=$ Measurement noise

$k=$ Kalman gain

The value of $\mathrm{R}$ in this paper is determined by the standard deviation of a sample of RSSI values and $\mathrm{Q}$ is half of the value of $\mathrm{R}$ to reduce time lag. The value of $\mathrm{Q}$ and $\mathrm{R}$ are tweaked during the offline testing phase.

\section{RESULTS AND ANALYSIS}

The experiment as explained in Section 2 is conducted. The resulting graphs and data are presented in this section. To observe the effect of the digital filters, four samples of 500 RSSI readings are obtained with the sampling rate of $10 \mathrm{~Hz}$ from receiver ESP8266. The result of the experiment is as shown in the graph of Figure 4.

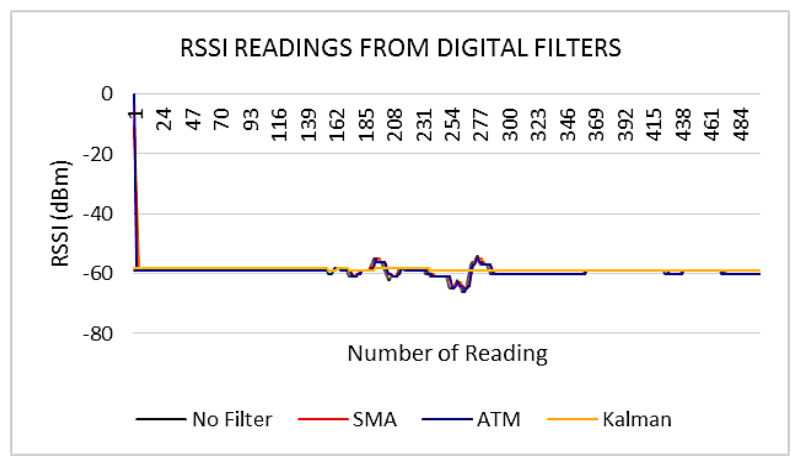

Figure 4. Received Signal Strength Indicator readings for no filter, SMA filter, ATM filter and Kalman filter

The RSSI readings obtained from the experiment are categorized under four conditions which are settling period, before crossing of a human subject, during the crossing of a human subject and after the crossing of a human subject. The period of each condition is as in Table 1.

Table 1. Category of Received Signal Strength Indicator Conditions

\begin{tabular}{ccccc}
\hline Reading & Settling Period & Before Crossing & During Crossing & After Crossing \\
\hline Start & 1 & 5 & 156 & 286 \\
End & 4 & 155 & 285 & 500 \\
\hline
\end{tabular}

\subsection{Noise reduction}

The noise reduction is observed by computing the standard deviation of the RSSI readings samples. Standard deviation is a measurement that quantifies the dispersion of data values. In this paper, the standard deviation value can indicate how close the RSSI readings are to its mean. Hence, the observation of the standard deviation value of the sample in this experiment is the best indicator of noises. It is safe to assume that the higher the value of the sample standard deviation, the higher the noises. The standard deviation values of the data samples are tabulated in Table 2. 
From the values observed in Table 2, Kalman filter is observed to have the least values of standard deviation throughout the three conditions which are 0 before crossing, 0.49 during crossing and 0.00 after crossing. On the other hand, ATM filter is observed to have the highest values of standard deviation throughout the three conditions which are 0 before crossing, 2.37 during crossing and 0.51 after crossing.

Table 2. Standard deviation of data samples

\begin{tabular}{cccc}
\hline \multirow{2}{*}{ Digital Filter } & \multicolumn{3}{c}{ Sample Standard Deviation, S } \\
& Before Crossing & During Crossing & After Crossing \\
\hline No Filter & 0 & 2.49 & 0.50 \\
SMA & 0 & 2.33 & 0.51 \\
ATM & 0 & 2.37 & 0.51 \\
Kalman & 0 & 0.49 & 0.00 \\
\hline
\end{tabular}

\subsection{Data proximity}

The data proximity is a criterion to observe how close the mean values of the filtered RSSI readings to the mean value of unfiltered RSSI readings. This is to assume how accurate the filtered readings are to its actual value. The higher the difference between the mean values of filtered RSSI readings and unfiltered RSSI readings, the further it is to the actual value. The mean values of the data samples are tabulated in Table 3. From the values observed in Table 3, Kalman filter is observed to have the highest difference in mean value throughout the three conditions which are 1 before crossing, 0.99 during crossing and 0.56 after crossing. On the other hand, ATM filter is observed to have the least difference in mean value throughout the three conditions which are 0 before crossing, 0.13 during crossing and 0.03 after crossing.

Table 3. Mean of data samples

\begin{tabular}{cccc}
\hline Digital Filter & Before Crossing & $\begin{array}{c}\text { Mean }(\mathrm{dBm}) \\
\text { During Crossing }\end{array}$ & After Crossing \\
\hline No Filter & -59 & -59.57 & -59.56 \\
SMA & -59 & -59.26 & -59.50 \\
ATM & -59 & -59.44 & -59.53 \\
Kalman & -58 & -58.58 & -59.00 \\
\hline
\end{tabular}

\subsection{Delays}

The delays observed in this paper are Settling Delay and Response Delay. Settling delay is the time taken for a filter to collect sufficient RSSI readings to produce an acceptable data. Response delay is the time taken for filtered readings to produce changes as affected by unfiltered input readings. The time delay can be calculated using equation 8 . The sampling rate used in the experiment of this paper is $10 \mathrm{~Hz}$.

$$
\text { Time Delay }=\frac{1}{\text { Sampling Rate }} \times \text { Number of Reading }
$$

Figure 5 shows the graph of 10 RSSI readings as sampled from the result in Figure 4 . It can be observed that the SMA filter has the highest settling delay by $0.5 \mathrm{~s}$ and Kalman filter has the least settling delay by 0s. To analyze the response delay, RSSI readings between the condition of before crossing and during the crossing of a human subject as sampled from the result in Figure 4 are observed. Figure 6 shows the graph of RSSI readings with SMA filter. Figure 7 shows the graph of RSSI readings with ATM filter. Figure 8 shows the graph of RSSI readings with Kalman filter.

It can be observed that SMA filter has the highest response delay by $0.6 \mathrm{~s}$ and Kalman filter has the least response delay by 0s. To envision the purpose of this analysis, an example of RSSI readings that is implemented in physical intrusion detection is discussed. In intrusion detection, the data collected during human crossing is considered to be very sensitive. From the graph of Kalman filtered RSSI readings in Figure 9, it can be observed that the noises during the crossing of a human subject (reading 156 to 285) are significantly smoothed where an intrusion is unlikely to be detected. Furthermore, there are no significant negative peak value that can be extracted during the period which make the data useless for a radio frequency signature identification. On the other hand, SMA filter may seem to be equally favourable to ATM filter. SMA filter has a slightly higher performance in noise reduction compared to ATM filter by $1.72 \%$. However, referring back to the criteria requirement of the implementation example, noise reduction is weighted lesser compared to delays. ATM filter has a higher performance in delays where the data is more responsive to 
changes as shown in graph of Figure 10. Higher data responsiveness will result in faster physical intrusion detection. Thence, ATM Filter is more efficient to be used in the pre-processing of RSSI readings for physical intrusion detection.

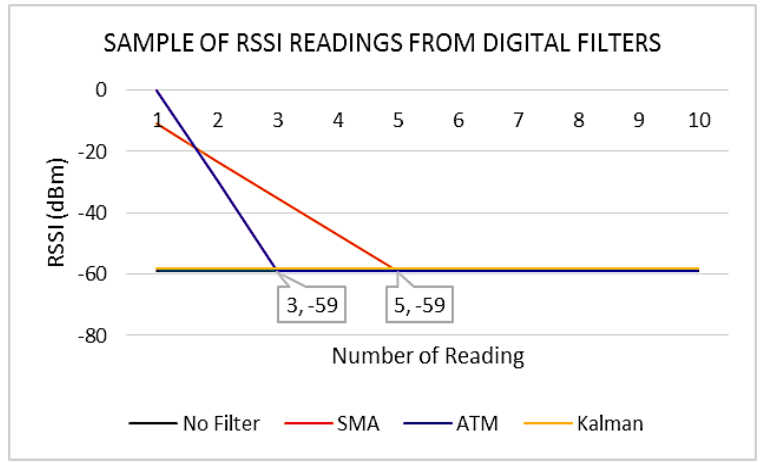

Figure 5. Graph of Received Signal Strength Indicator readings from reading 1 to reading 10 as sampled from readings in graph of Figure 4

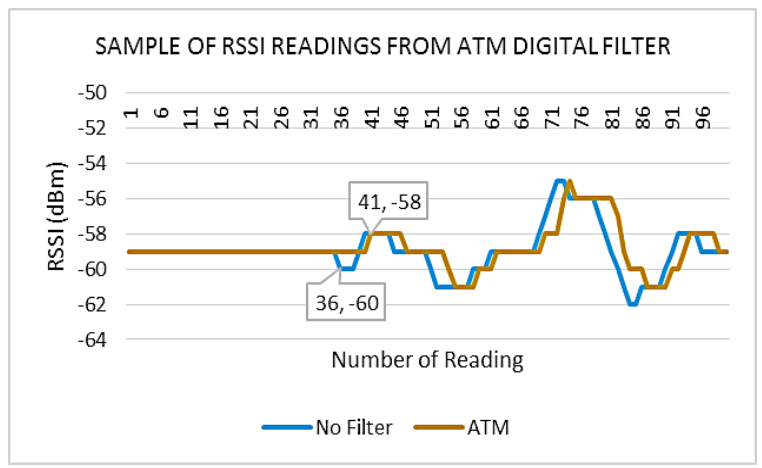

Figure 7. Graph of Received Signal Strength Indicator readings with ATM filter

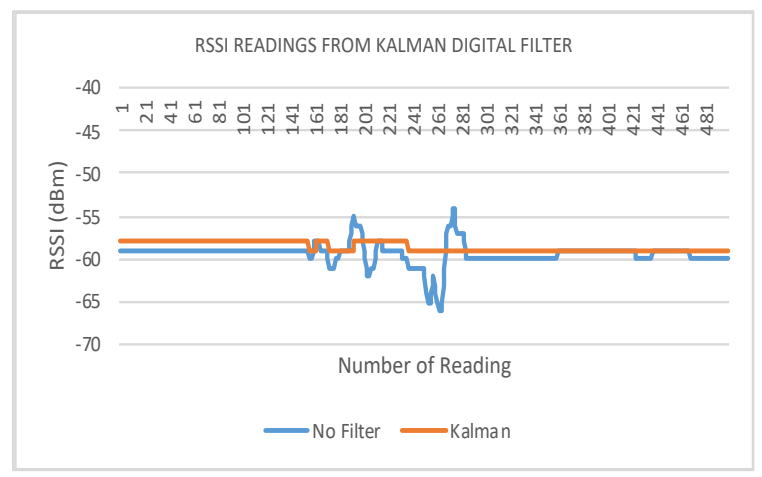

Figure 9. Graph of Received Signal Strength Indicator readings with Kalman filter

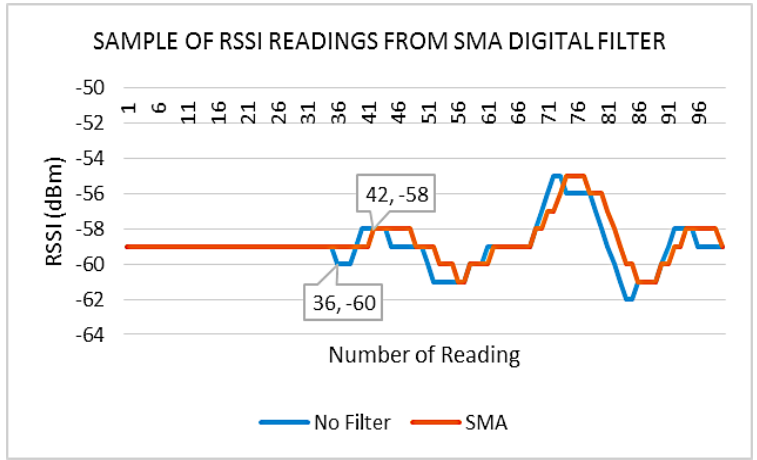

Figure 6. Graph of Received Signal Strength Indicator readings with SMA filter

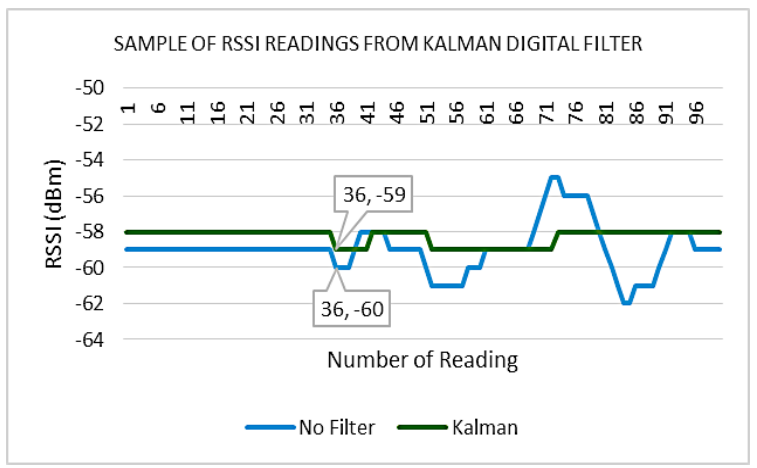

Figure 8. Graph of Received Signal Strength Indicator readings with Kalman filter

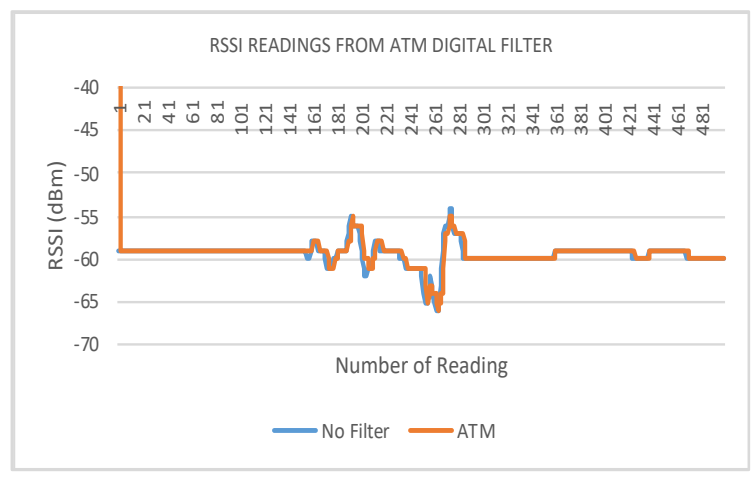

Figure 10. Graph of Received Signal Strength Indicator readings with Alpha-Trimmed Mean filter

\section{CONCLUSION}

The comparison analysis of three different digital filters for RSSI readings has been carried out and presented in this paper. There are many options for digital signal processing filters that can be used. However, the choice of digital signal processing filters has to be aligned with the implementations and 
applications. In this paper, there are three performance evaluation criteria were observed during the experiment. They are noise reduction, data proximity and delays. The results have shown that the filter with the highest performance in noise reduction is Kalman filter followed by SMA filter and lastly ATM filter. Secondly, the filter with the highest performance in data proximity is ATM filter followed by SMA filter and lastly Kalman filter. Finally, the filter with the highest performance in delays is Kalman filter followed by ATM filter and lastly SMA filter. All the filters are highly beneficial subjected to its implementation. For example, ATM Filter is more efficient to be used in the pre-processing of RSSI readings for physical intrusion detection due to its high data proximity and responsiveness. Hence, the paper recommends that depending on thee RSSI application, a specific digital filter may be used individually oer in combination with different other filters.

\section{ACKNOWLEDGEMENTS}

The research done in this paper is partially funded by the Malaysian Ministry of Higher Education (MOHE) under Prototype Development Research Grant (PRGS), PRGS16-009-0040 and IIUM Publication RIGS grant no. P-RIGS19-003-0003.

\section{REFERENCES}

[1] O. G. Adewumi, K. Djouani and A. M. Kurien, "RSSI based indoor and outdoor distance estimation for localization in WSN," 2013 IEEE International Conference on Industrial Technology (ICIT), Cape Town, 2013, pp. 1534-1539.

[2] S. J. Halder, P. Giri and W. Kim, "Advanced smoothing approach of RSSI and LQI for indoor localization system," International Journal of Distributed Sensor Networks, 11(5), 195297, 2015.

[3] M. Ben Kilani, A. J. Raymond, F. Gagnon, G. Gagnon and P. Lavoie, "RSSI-based indoor tracking using the extended Kalman filter and circularly polarized antennas," 2014 11th Workshop on Positioning, Navigation and Communication (WPNC), Dresden, 2014, pp. 1-6.

[4] L. Khalil and P. Jung, "Scaled Unscented Kalman Filter for RSSI-based Indoor Positioning and Tracking," 2015 9th International Conference on Next Generation Mobile Applications, Services and Technologies, Cambridge, 2015, pp. 132-137.

[5] Z. Jianyong, L. Haiyong, C. Zili and L. Zhaohui, "RSSI based Bluetooth low energy indoor positioning," 2014 International Conference on Indoor Positioning and Indoor Navigation (IPIN), Busan, 2014, pp. 526-533.

[6] M. Botta and M. Simek, "Adaptive distance estimation based on RSSI in 802.15. 4 network," Radioengineering, 22(4), 1162-1168, 2013.

[7] W. W. Li, R. A. Iltis and M. Z. Win, "A smartphone localization algorithm using RSSI and inertial sensor measurement fusion," 2013 IEEE Global Communications Conference (GLOBECOM), Atlanta, GA, 2013, pp. 3335-3340.

[8] V. Malyavej, W. Kumkeaw and M. Aorpimai, "Indoor robot localization by RSSI/IMU sensor fusion," 2013 10th International Conference on Electrical Engineering/Electronics, Computer, Telecommunications and Information Technology, Krabi, 2013, pp. 1-6.

[9] Y. Lee and S. Park, "RSSI-based fingerprint map building for indoor localization," 2013 10th International Conference on Ubiquitous Robots and Ambient Intelligence (URAI), Jeju, 2013, pp. 292-293.

[10] Yiu, S., Dashti, M., Claussen, H., \& Perez-Cruz, F. "Wireless RSSI fingerprinting localization”. Signal Processing, 131, 235-244. 2017.

[11] R. S. Rosli, M. H. Habaebi and M. R. Islam, "Comparative Analysis of Digital Filters for Received Signal Strength Indicator," 2018 7th International Conference on Computer and Communication Engineering (ICCCE), Kuala Lumpur, 2018, pp. 214-217.

[12] R. S. Rosli, M. H. Habaebi and M. R. Islam, "Characteristic Analysis of Received Signal Strength Indicator from ESP8266 WiFi Transceiver Module," 2018 7th International Conference on Computer and Communication Engineering (ICCCE), Kuala Lumpur, 2018, pp. 504-507.

[13] “ESP-01 WiFi Module,” PDF, AI-Thinker team, Version1.0, 2015.

[14] M. Habaebi, R. Rosli, \& M. R. Islam, "RSSI-based Human Presence Detection System for Energy Saving Automation," Indonesian Journal of Electrical Engineering and Informatics (IJEEI), 5(4), 339-350, 2017.

[15] Agel, M.M., Habaebi, M.H., Islam, M.R., "Mitigation of multipath fading in indoor radiometric fingerprinting systems", Elsevier Computers and Electrical Engineering, 73, pp. 46-57, January 2019. 


\section{BIOGRAPHIES OF AUTHORS}

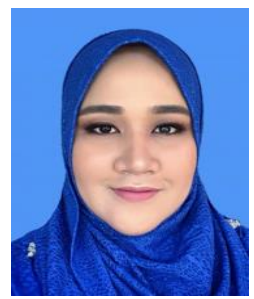

Rafhanah Shazwani Rosli is a Graduate Research Assistant at the department of Electrical and Computer Engineering, International Islamic University Malaysia (IIUM). She obtained her Bachelor of Engineering in Electronic - Computer \& Information from International Islamic University Malaysia in 2016. She is currently doing her Master of Science in Computer and Information Engineering at International Islamic University Malaysia. Her current research is in the domain of Internet of Things.

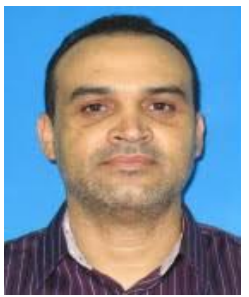

Mohamed H. Habaebi (M'99-SM'16) received his first degree from the Civil Aviation and Meteorology High Institute (CAHI), Libya ('91), his MSc in Electrical Engineering from Universiti Teknologi Malaysia ('94), and his Ph.D. in Computer and Communication System Engineering from Universiti Putra Malaysia ('01). He is currently an Associate Professor and the Post Graduate Academic Advisor at the Department of Electrical and Computer Engineering, International Islamic University Malaysia. He heads the research works on Internet of Things at the department. He has supervised many Ph.D. and M.Sc. students, published more 120 articles and papers and sits on editorial board of many international journals. He is actively publishing in M2M communication protocols, wireless sensor and actuator networks, cognitive radio, small antenna system \& radio propagation and wireless communications \& network performance evaluation. He is an active member of IEEE and an active reviewer to many international journals.

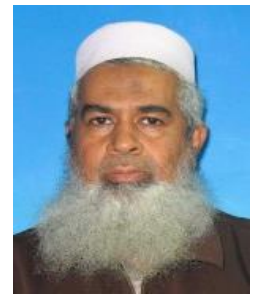

Md Rafiqul Islam (M'02) received his Bachelor of Science in Electrical and Electronic Engineering from Bangladesh University of Engineering \& Technology (BUET), Dhaka in 1987. $\mathrm{He}$ received his MSc and $\mathrm{PhD}$ both in Electrical Engineering from University of Technology Malaysia in 1996 and 2000 respectively. He is currently working as a professor in the Department of Electrical and Computer Engineering, Faculty of Engineering, International Islamic University Malaysia. He has supervised more than $50 \mathrm{PhD}$ and MSc students and has published more than 200 research papers in international journals and conferences. His areas of research interest are wireless channel modeling, radio link design, RF propagation measurement and modeling in tropical and desert, RF design, smart antennas and array antennas design, FSO propagation and modeling etc. He is a Life Fellow of Institute of Engineers Bangladesh and member of IEEE and IET. 